\title{
Analysing User Satisfaction in Next Generation Networks for Multimedia Multicast Transmission
}

\author{
Iffat Ahmed, Andreas Petlund \\ Simula Research Laboratory, University of Oslo, Norway \\ E-mail: $\{$ iffat, apetlund $\} @$ simula.no
}

\begin{abstract}
Video users in a multicast group may be highly heterogeneous in terms of individual channel conditions and requirements for video transmission, making it a challenging task for the network to optimally configure the resource management. In this paper, we consider a mathematical model for the choice of the optimum number of transmission opportunities, based on a Markov chain representation of the wireless channel, where each channel state is associated to a different quality corresponding to a choice of video layer and Modulation and Coding Scheme (MCS). In case of multicast transmissions, the selection of the video layer is based on the aggregate channel conditions of all users and a collection of coordination rules. We evaluate how the cross-layer optimisation performs and also define a taxonomy of user quality perception. We further investigate how users' satisfaction levels vary in the multicast session. Performance evaluation results show that cross-layer optimisation significantly outperforms sequential and independent scheme for multicast video transmissions, conforming the strong need for cross-layer solutions in video transmission, however, this improvement is heavily related to the resource allocation policy of the operator.
\end{abstract}

Index Terms-Video; multicast; user satisfaction; channel rate

\section{INTRODUCTION}

Next Generation Networks are facing a continued increase in usage of multimedia applications, such as video conferencing and video streaming. This has led to an increased demand for bandwidth and also implies a big challenge for network operators to properly utilise network resources and provide adequate quality to users when faced with heterogeneous terminals and network infrastructures. Well known documents like the Cisco report [1] offer evidence of such a "video explosion". They predict that the amount of video content crossing global IP networks each month in year 2016 will amount to the equivalent of over 6 million years of video playout.

Research on video services usually only consider point-topoint scenarios; less investigation has been done for multicast scenarios. Similarly, many studies have been performed to improve the quality of transmitted video by using mechanisms for efficient resource allocation [3], congestion control [4] or bandwidth/rate management [5]. Multicast communication is an important solution for distributed multimedia applications, particularly video, to efficiently use the network resources by exploiting spatial and content redundancy of the user requests. Multimedia applications are sensitive to latency, and can be resource intensive. Proper network resource management may help avoid situations where users experience a broken service. Combining multicast with knowledge about video playout choices may help achieve such resource management.
Among the different characteristics of video traffic, we particularly focus on its layered structure, with particular reference to the H.264 Scalable Video Coding (SVC) [6], [7] an extension of the H.264 Advance Video Coding (AVC), in which the bit stream is divided into layers: one Base Layer (BL) providing basic video quality and multiple Enhancement Layers (ELs) bringing additional incremental quality. ELs depend on the BL, since they cannot be decoded unless BL is fully decoded first. As long as the users receive SVC base layer content, they are able to decode the video, and when they receive SVC enhancement layers, the quality of the video is increased.

Future wireless networks will exploit improved data rates offered by the ability to closely follow the channel dynamics [8]. High-order modulations can improve the spectral efficiency of the transmission. They can, however, also increase the bit error probability, which is undesirable for video contents given their specific error sensitivity.

The incremental structuring of the content may cause error propagation in the following video content, and therefore unacceptable quality fluctuations and artefacts at the end users may occur. Thus, Adaptive Modulation and Coding (AMC) schemes are required to achieve high data rates. Due to the diverse channel conditions at the end users, we can adaptively select channel rates and select the layer of the SVC source accordingly.

This problem gets more important in the multicast case. Here, instead of looking into individual channel conditions and the packets of the available video stream for the single user, the assignment of base layer and enhancement layers must be coordinated among all the users in the multicast group. Thus, a suitable trade-off must be found among their channel conditions, and their diverse video quality requirements.

At a first glance, these problems are similar to general packet scheduling frameworks that have been proposed in the literature [9], [10]. However, while this kind of resource allocation has been deeply explored, it was often targeting Quality of Service (QoS) provisioning, which usually refers to improving goodput (i.e., the net fraction of throughput after removing coding overhead), link utilisation and packet delay, but it does not necessarily improve the end user perceived quality.

Also, [6] investigates how enhancement layers of a SVC bit stream can improve the perceived user QoE. That paper shows that if the relationship between BL and ELs is properly accounted for in the resource allocation scheme, the resulting 
video quality is considerably increased compared to the use of the H.264-AVC or MPEG-4 codecs; however, wireless channel models are not considered. A resource allocation scheme for scalable video has been presented by [11], which allots the modulation coding according to the video layer to be transmitted, providing an efficient allocation for the timeslots. The proposed solution is compared to a single layer video with fixed modulation and coding scheme.

To improve the quality of multicast streaming for adaptive video, a cross-layer architectural solution is proposed in [12], which incorporates signalling between PHY, MAC and application layers and tries to improve delay, jitter and packet loss. At the same time, the amount of required overhead is investigated, seeking for a compromise on the signalling increase due to cross-layer information exchange. Another similar approach is that of [13], which uses dynamic adaptive streaming with SVC to provide a cost effective solution. It focuses on improving the video segment quality according to the user download rate. The research variables considered are quality variation, performance, truncated normal rate, and so on, but there is no consideration on a wireless channel model nor a multicast analysis, as the solution focuses on a single generic transmission between the client and the server. A utility (representing QoE) maximisation problem is considered by [14], which focuses on user-dependent and session dependent utility functions. It tries to allocate resources efficiently to multicast groups and investigates the performance over average user rate, based on the number of groups. Even though it analyses the multicast user performance, it does not investigate packet differentiation into BL and EL packets, as we do.

Instead, all the procedures that we perform to specifically address video content target the improvement of the Quality of Experience (QoE), i.e., how the end users perceive their received video content. To understand the difference between a pure QoS and QoE enhancement, we consider the case where a good channel quality is exploited for transmitting several enhancement layer packets. While the goodput is improved, and therefore the QoS, the resulting quality may be totally unaffected if the base layer is not correctly received. This is why we advocate a PHY/Application cross-layer optimisation, not only a generic "cross-layer" optimisation, even though it is challenging as it has to span the entire protocol stack. The terms goodput, quality and delay are used hereafter with a QoE awareness; specifically taking QoE of video into account.

To investigate the performance of the PHY/Application cross-layer optimisation we consider an analytical model, where the channel is modelled by means of a Markov chain, whose states represent different channel qualities. This problem is addressed first in a unicast scenario [15], then extended to a multicast scenario, where the resource allocation becomes a challenging trade-off among the different needs of the users.

To sum up, in this paper we make these contributions:

i) Is PHY/Application cross-layer optimisation really required for video transmission over next generation wireless networks? Or would a sequential allocation where optimisation is independently performed at the PHY and Application layers work similarly?

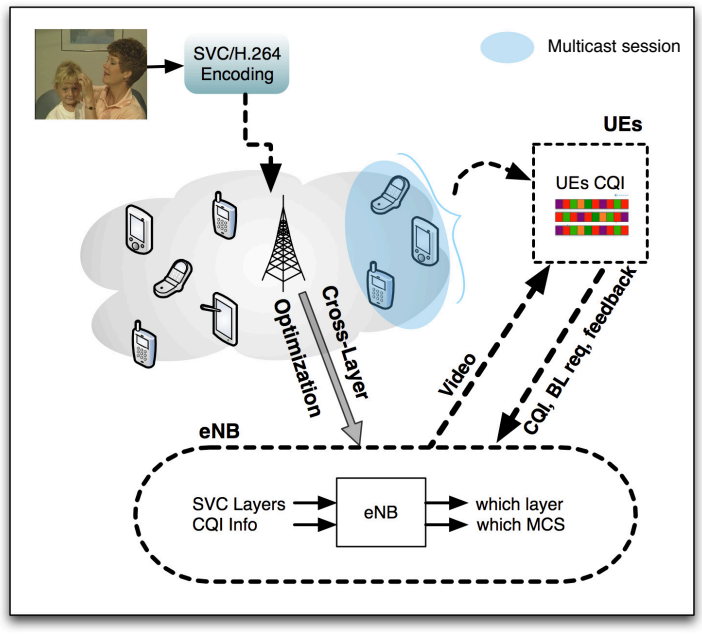

Fig. 1: Multicast Scenario

ii) How do cross-layer and non-cross-layer optimisations perform also compared to the theoretical best allocation that one could apply, if the channel states and the user quality requirements were all known in advance? Is there a way to adapt to the channel variability?

iii) How does the unicast scenario extend to the multicast case? The latter scenario demands to seek a compromise in resource allocation between the needs of all the users, and we will check if cross-layer optimisation is helpful even in this case.

The paper is organised as follows. Section II defines the system model, scenarios, and policies. Numerical performance evaluations are presented in Section III via an extensive simulation campaign. Finally, Section IV discusses the main findings and concludes the paper.

\section{ANALYTICAL MODEL}

Taking advantage of adaptive channel rates and scalable video layers for quality enhancement can significantly improve the perceived quality experienced by the users. For this reason, we try to exploit both features to provide enhanced QoE for scalable video while taking into consideration varying channel conditions of the individual users. For simplicity, we just assume two layers, i.e., one enhancement layer beyond the base layer. The extension to multiple ELs would be conceptually straightforward. Thereafter, indices "1" and "2" refer to the base and enhancement layers, respectively. The notation used throughout this work is presented in Table I.

This section mathematically models the choice of the optimal number of transmit opportunities (TXOPs) required for the users to decode the base layer and maximise their perceived quality in unicast and multicast environments. Since, each user has its BL and EL requirement, we consider it as user expectation and eventually what user has received is called user perception in our model. Therefore, based on this expectation and perception of the user, we discuss the user satisfaction and define user taxonomy. We consider a fixed number of TXOPs, since this supposedly models the real time characterisation of video (after $T$ timeslots, we start 
TABLE I: Notations and assumptions

\begin{tabular}{|c|c|}
\hline Paramter & Description \\
\hline$T$ & Total number of $k$ time slots \\
\hline$N$ & Number of users $j \in N$ \\
\hline $\mathcal{L}, \mathcal{Z}, \mathcal{S}$ & $\begin{array}{l}\text { Set of SVC layers, MCS and Channel States, } \\
\text { respectively }\end{array}$ \\
\hline$\zeta, \ell$ & Generic elements of $\mathcal{Z}$ and $\mathcal{L}$ \\
\hline$t_{1}, t_{2}$ & $\begin{array}{l}\text { Number of time slots for a base and enhancement } \\
\text { video layer, respectively; note that } t_{1}+t_{2}=T\end{array}$ \\
\hline$p_{j}, P_{j}^{d}$ & $\begin{array}{l}\text { Packet loss and decoding probability of user } j \text {, } \\
\text { respectively }\end{array}$ \\
\hline$\theta_{j}$ & $\begin{array}{l}\text { Threshold for base layer packets that each user } j \\
\text { must receive to correctly decode the stream }\end{array}$ \\
\hline$\hat{g}_{j}$ & Expected goodput of user $j$ \\
\hline$\hat{n}_{1}, \hat{n}_{2}$ & $\begin{array}{l}\text { Requested number of BL and EL packets, respec- } \\
\text { tively }\end{array}$ \\
\hline$n_{1}, n_{2}$ & $\begin{array}{l}\text { Received number of BL and EL packets, respec- } \\
\text { tively }\end{array}$ \\
\hline$g_{\ell, \zeta}^{k}, d_{\ell, \zeta}^{k}, q_{\ell, \zeta}^{k}$ & $\begin{array}{l}\text { Goodput, packet delivery delay and quality with } \\
\operatorname{MCS} \zeta \text { and SVC layer } \ell \text { at time } k \text {, respectively }\end{array}$ \\
\hline$\gamma$ & $\begin{array}{l}\text { Weight of the linear combination of goodput and } \\
\text { delay }\end{array}$ \\
\hline$G_{j}, D_{j}, Q_{j}$ & $\begin{array}{l}\text { Goodput, packet delivery delay and quality of the } \\
\text { user } j \text {, respectively }\end{array}$ \\
\hline$\chi_{a v g}, \chi_{\max }$ & $\begin{array}{l}\text { Number of BL packets computed as multicast value } \\
\text { using Average and Maximum strategy, respectively }\end{array}$ \\
\hline$\alpha, \beta$ & $\begin{array}{l}\text { The weight for BL and EL quality assessment, } \\
\text { respectively }\end{array}$ \\
\hline$\lambda$ & Fraction of transmissions reserved for $\mathrm{BL}$ \\
\hline
\end{tabular}

with new video contents periodically). Finally, the framework also describes the network policies evaluated in the subsequent sections.

\section{A. Taxonomy of Users}

To categorise the user satisfaction about the perceived video quality, we define the following taxonomy of the users' statuses with respect to whether their QoE requirements (in particular, decoding of the flow) are met.

Satisfied - Within the current batch of video content transmission, a user $j$ is said to be satisfied, if it has received enough base layer packets to decode the video within the available number of transmit opportunities, i.e., $n_{1}(j) \geq \theta_{j}$.

Happy - A user is said to be happy, if it expects that it will eventually receive the required number of base layer packets to decode the video within the available transmit opportunities. Whenever this expectation is confirmed, this user will become satisfied. Conversely, if the expectation is contradicted, the user will become unhappy or hopeless.

UnHappy - A user is said to be unhappy, if it will likely not receive the required number of base layer packets to decode the video before the end of the available transmit opportunities. Similarly, confirmation of this expectation leads the user to become Hopeless or the expectation can change to Happy or Satisfied.

Hopeless - User $j$ is said to be Hopeless if it is sure that it will never be satisfied within the available number of transmit opportunities. That is, it will not receive the required number of base layer video packets to decode correctly within the available transmit opportunities, i.e., $n_{1}(j)(T)<\theta_{j}$.

\section{B. Mathematical Model Scenarios}

For the sake of simplicity, we assume an independent (i.i.d) error process on the channel. Therefore, the probability to correctly receive a packet is $1-p_{j}{ }^{1}$. The decoding probability of the flow for user $j$ corresponds to the probability of decoding $\theta_{j}$ packets out of $t_{1}$ TXOPs, i.e.

$$
P_{j}^{d}= \begin{cases}0 & \text { if }\left(1-p_{j}\right) \cdot n_{1}<\theta_{j} \\ F & \text { if }\left(1-p_{j}\right) \cdot n_{1} \geq \theta_{j}\end{cases}
$$

where, in first case, if the user does not received enough BL packets so that it cannot decode the video, therefore, quality is zero, and $F$ is defined as

$$
F=\sum_{k=0}^{t_{1}-\theta_{j}}\left(\begin{array}{c}
t_{1} \\
k
\end{array}\right)\left(1-p_{j}\right)^{t_{1}-k} p_{j}{ }^{k}
$$

We formalize the expected goodput of user $j$ as:

$\hat{g}_{j}= \begin{cases}0 & \text { if non-decoding } \\ \alpha \cdot \hat{n}_{1}(j)\left(1-p_{j}\right)+\beta \cdot \hat{n}_{2}(j)\left(1-p_{j}\right) & \text { if decoding }\end{cases}$

where $\alpha$ and $\beta$ are properly chosen weights that regulate the relative importance of BL packets versus enhancement layer packets in terms of supplied quality, therefore, $\beta>\alpha$ to give more weight to EL. Thus, the average perceived goodput is

$$
\begin{aligned}
\mathbb{E}\left[\hat{g}_{j}\right] & =\left(\alpha t_{1}\left(1-p_{j}\right)+\beta t_{2}\left(1-p_{j}\right)\right) \\
& \times \sum_{k=0}^{t_{1}-\theta_{j}}\left(\begin{array}{c}
t_{1} \\
k
\end{array}\right)\left(1-p_{j}\right)^{t_{1}-k} p_{j}{ }^{k} u\left[t_{1}-k\right]
\end{aligned}
$$

where $u[x]$ is a unit step, i.e., it is one if $x$ is non-negative, zero otherwise.

It is straightforward to identify the optimum value of $t_{1}$ that maximizes the expected quality as per (4) from an a priori standpoint. Therefore, we define $\operatorname{Opt}_{t_{1}}(j)$ as the value of $t_{1}$ between 0 and $T$ that provides maximum expected quality for user $j$. If we have multiple users, all with identical error probability $p_{j}$, we can derive the best policy for the operator, i.e., the optimal split of $T$ into $t_{1}$ and $t_{2}$ so that the quality according to (3) is maximized. Thus, formally

$$
O p t_{t_{1}}(j)=\arg \max \mathbb{E}\left[\hat{g}_{j}\right]
$$

subject to

$$
\begin{aligned}
\left(1-p_{j}\right) \cdot t_{1} & \geq \theta_{j} \\
T & =t_{1}+t_{2}
\end{aligned}
$$

We can write the former constraint as $\left(1-p_{j}\right) \cdot t_{1}-\theta_{j} \geq$ 0 , and latter as $t_{1}+t_{2}-T=0$. Further, if the function to be maximized is denoted as $f$, and the above mentioned constraints as $g$ and $h$, we have the following goal function and constraints:

$$
\begin{aligned}
f\left(t_{1}, t_{2}, p_{j}\right) & =\left(\alpha t_{1}\left(1-p_{j}\right)+\beta t_{2}\left(1-p_{j}\right)\right) \\
& \times \sum_{k=0}^{t_{1}-\theta_{j}}\left(\begin{array}{c}
t_{1} \\
k
\end{array}\right)\left(1-p_{j}\right)^{t_{1}-k} p_{j}^{k} u\left[t_{1}-k\right] \\
g\left(t_{1}, t_{2}, p_{j}\right) & \geq\left(1-p_{j}\right) \cdot t_{1}-\theta_{j} \\
h\left(t_{1}, t_{2}, p_{j}\right) & =t_{1}+t_{2}-T
\end{aligned}
$$

${ }^{1}$ For simplicity, we use the same $p_{j}$ for BL and EL packets, but the model can be extended to the more realistic case where BL and ELs have different levels of error protections 
We can utilize the Lagrange Multiplier optimization method to find the maxima [16], so that we can find the additional number of timeslots required to correctly decode the video as

$$
\eta=\frac{\beta p_{j} T}{(\beta-\alpha)}
$$

Now the optimum $t_{1}$ would be equal to $\theta_{j}$ plus the additional number of transmit opportunities as in (9) required to successfully decode:

$$
O p t_{t_{1}}=\theta_{j}+\eta
$$

We expand the analysis to a scenario in which each user can receive from 0 to $n$ packets at each transmit opportunity, according to its loss probability.

We assume the number of received packets is Bernoulli distributed, related to the individual loss probability. Based on the loss probability $p_{j}, n$ probabilities are calculated, $\left\{\rho_{0}(j), \rho_{1}(j), \rho_{2}(j) \ldots \rho_{n}(j)\right\}$ according to the Bernoulli Distribution. The decoding probability $P_{j}^{d}$ becomes

$$
P_{j}^{d}= \begin{cases}0 & \text { if } t_{1} \sum_{i=0}^{n} i \rho_{i}(j)<\theta_{j} \\ g_{j} & \text { if } t_{1} \sum_{i=0}^{n} i \rho_{i}(j) \geq \theta_{j}\end{cases}
$$

where $g_{j}$ is the expected quality for user $j$, defined as

$$
\mathbb{E}\left[g_{j}\right]=\alpha t_{1}\left(\sum_{i=0}^{n} i \rho_{i}(j)\right)+\beta\left(T-t_{1}\right)\left(\sum_{i=0}^{n} i \rho_{i}(j)\right)
$$

Similar to (5), we can find the optimum value of $t_{1}$ satisfying, $\nu_{j} t_{1}>\theta_{j}$ and $t_{1}<T$, and which provides the maximum expected quality given $P_{j}^{d}$ for user $j$ :

$$
O p t_{t_{1}}(j)=\arg \max \mathbb{E}\left[\hat{g}_{j}\right]
$$

To better analyze the tradeoff between various policies, we define $\lambda$ as a threshold for a minimum number of BL packets required by user $j$, after which various policies (defined in Section II-C) behave differently. We varied $\lambda$ for different values in the range $[0,1]$ to investigate the impact of policies differentiation. Thus, for each user $j$ we define a minimum number $\chi_{j}$ of BL and after this value, the base station allocates resources with the choice of BL/EL packets and MCS. Thus, the cross-layer and non-cross-layer approaches behave differently. In unicast, the base station allocates resources according to the individual needs of user $j$ depending upon its own $\chi_{j}$, therefore

$$
\chi_{j}=O p t_{t_{1}}(j) \times \lambda
$$

Hence, $\lambda$ is a threshold defining the point after which we can allocate video packets (either BL or EL) according to three allocation policies (defined in Section II-C). For example, $\lambda=0.5$ means we restrict the system to transmit BL packets $50 \%$ of the time, after which the system has the choice to transmit BL or EL packets depending on the user's need and channel quality.

Note that in general each user will have a different optimal $t_{1}$-value, that is each user $j$ has its own $O p t_{t_{1}}$, therefore the operator needs to find a suitable tradeoff among the needs of all the served users for multicast sessions.

Multicast Scenario - Once each user (UE) has requested its required number of transmit opportunities to the central base station (eNB), and the individual optimal $t_{1}$ of each user is found, then based on various strategies, the base station decides the optimum number of transmit opportunities to transmit BL and EL for all the users in the multicast group as in Figure 1. In this work, we take into consideration the following two strategies. Note that in the multicast transmission, the base station has to consider the individual channel conditions of all the users, and to consider aggregate channel conditions for the transmission of the same video contents to all the users.

Average Strategy - In this case, the base station computes the average $A v g_{-} O p t_{t_{1}}$ of all values $\mathrm{Opt}_{t_{1}}(j)$, and then transmits $\chi_{\text {avg }}$ packets from the base layer, the rest are enhancement layer packets. Therefore, for all the users, based on (10), the optimum number of timeslots for BL transmission will be computed as follows:

$$
A v g_{-} O p t_{t_{1}}=\frac{1}{N} \sum_{j=1}^{N} O p t_{t_{1}}(j)
$$

Some users will be served with fewer base layer packets than their requested $\hat{n}_{1}$, and some of them can even be unable to decode the stream. This strategy focuses on the quality improvements. Similar to the unicast case, the minimum threshold (regarding the number of BL received packets) to decode video in the multicast group based on $\chi_{\text {avg }}$, can be defined as $\chi_{\text {avg }}=A v g \_O p t_{t_{1}} \times \lambda$

Maximum Strategy - The users declare their preferred $\hat{n}_{1}$ and the central base station takes a conservative approach, selecting the largest $\hat{n}_{1}$ as the maximum of the declared values, to satisfy all the users. With this strategy, all the users may reasonably expect to be satisfied in the end, thus it tries to focus on fairness for all users. Therefore, at the beginning of the transmission they are all happy as defined in II-A. However, this strategy may decrease the QoE because more base layer and fewer enhanced layer packets will be received by all the users.

$$
\text { Max_Opt } t_{t_{1}}=\max _{j \in N}\left(O p t_{t_{1}}(j)\right)
$$

The minimum threshold (regarding the number of BL packets received) to decode video in the multicast group based on $\chi_{\max }$, can be defined as $\chi_{\max }=$ Max_Opt $t_{t_{1}} \times \lambda$

\section{Allocation Policies}

We design three allocation policies (the algorithms of these policies are described in detail in [15]): (i) a theoretical upper bound on the performance using a genie-like channel knowledge and offline optimisation, denoted as Offline; (ii) a sequential selection with optimisations performed separately at PHY and application layer, denoted as $N X L O$; (iii) a joint cross-layer optimisation of both PHY and application layers, denoted as $X L O$. Our goal is to investigate the cross-layer optimisation particularly for a SVC video source, it may be that cross-layer optimisation is insufficient, however we will check if this is the case as compared to non-cross layer optimisation.

\section{AnAlytical And Simulation Results}

The performance of the aforementioned policies is assessed by means of simulation in Matlab. Cross-layer resource management of video content is performed for $T$ timeslots. Table II presents the simulation parameters used (if not specified 


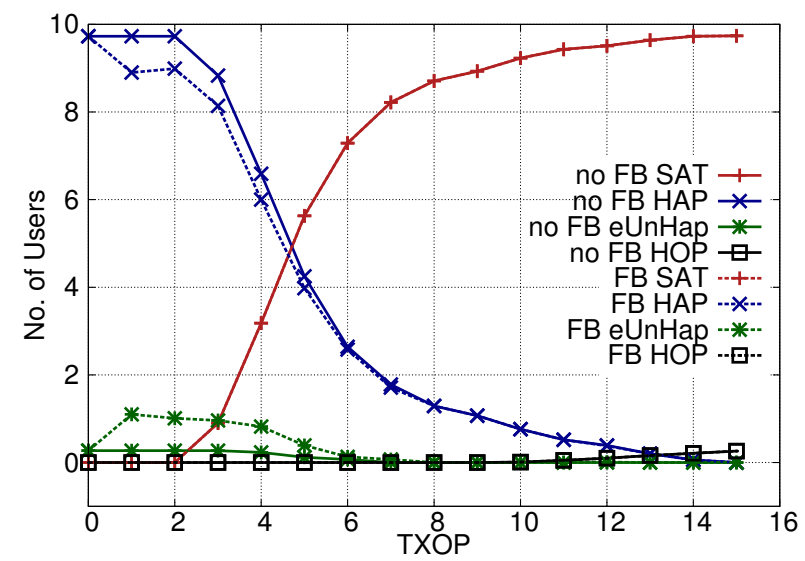

(a) Average strategy

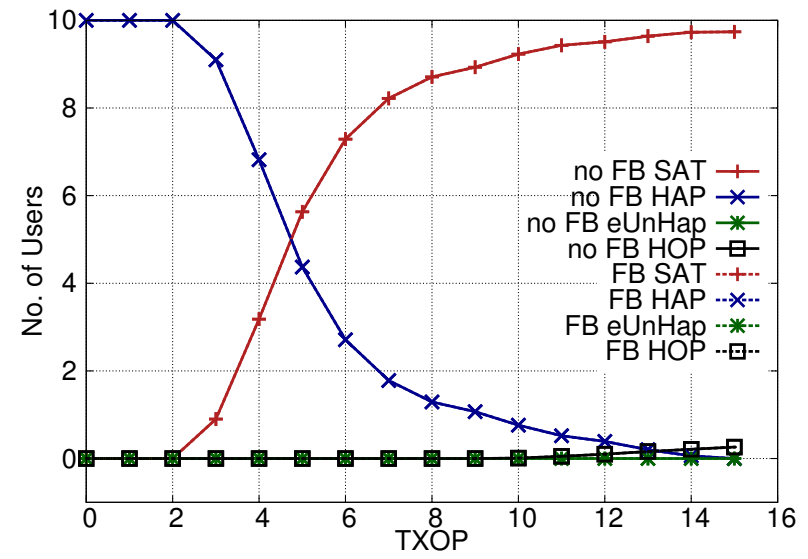

(b) Maximum strategy

Fig. 2: User Satisfaction Comparison for Average and Maximum strategy using feedback case with $T=15$. The dotted curve refer to the scenario where feedback (FB) mechanism is available and the solid lines represent results without feedback (noFB)

TABLE II: Simulation Parameters

\begin{tabular}{llll}
\hline \hline Param & Values & Param & Values \\
\hline$T$ & 100 & $\mathcal{L}$ & $\{1$-BL, 1-EL $\} \rightarrow\{0,1\}$ \\
$N$ & 10 & $\mathcal{Z}$ & $\{$ QPSK, 64-QAM $\} \rightarrow\{0,1\}$ \\
$\gamma$ & 0.6 & $\mathcal{S}$ & $\left\{\mathcal{S}_{00}, \mathcal{S}_{01}, \mathcal{S}_{10}, \mathcal{S}_{11}\right\}$ \\
$\alpha$ & 1 & $\lambda$ & $\left\{0, \frac{1}{4}, \frac{1}{2}, \frac{3}{4}, 1\right\}$ \\
$\beta$ & 5 & & \\
\hline
\end{tabular}

otherwise). Based on the varying channel conditions over time and the adopted scheduling policy, each user will get a certain number of BL and EL packets. Multiple users receive unicast video streams in parallel. This is coordinated by the base station when the same video is broadcast to all users in a multicast scenario. We first analyze the satisfaction level of the users depending on the availability of feedback mechanism. We further discuss the results for cross-layer optimisation versus non-cross-layer optimisation techniques and the impact of a multicast transmission for scalable video goodput, packet delivery delay and quality.

\section{A. Management Policies Scenarios and Results}

We simulate the system for different values of $T$. As before, each user has a random loss probability with uniform distribution between $0.01 \%$ and $1 \%$. There are four possible combinations based on the choice between Average and Maximum strategies, and including the availability of feedback in multicast environment.

Average strategy vs Maximum strategy - In the Average strategy, the base station averages all $O p t_{t_{1}}(j)$ values required by each user and then selects the mean value $\chi_{a v g}$ for all the users. For the Maximum strategy instead, the maximum value amongst $O p t_{t_{1}}(j)$ for all users is selected as $\chi_{\max }$ for multicast session. Even though the strategy is meant to be conservative, due to the chosen value of $\chi_{\max }$. some of the users may still be Hopeless or Unhappy, because, it may be that the number of available timeslots are not enough to cater the high requirements (regarding the number of BL packets) of the users.

Feedback vs Non-feedback cases - In the non-feedback case, the base station cannot update the $O p t_{t_{1}}(j)$ at each timeslot; therefore, it will assume the same $O p t_{t_{1}}(j)$ values and the computed $\chi_{a v g}$ or $\chi_{\max }$ which is initially calculated, if using the Average or Maximum strategy, respectively.

When feedback from each user is sent after each timeslot about the number of packets received and the perceived quality, the base station updates $O p t_{t_{1}}(j)$. In fact, thanks to the feedback, the $O p t_{t_{1}}(j)$ is updated at each transmission and the optimum value is selected again.

End-user Satisfaction - We present the results on the user satisfaction (Sec. II-A) based on the availability of a feedback mechanism. Fig. 2 represents the user satisfaction with $T$ set to 15, using Average and Maximum Strategies. In Fig. 2(a), the expected happy users (labeled as "HAP") at the beginning of the transmission are closely related to the actual number of satisfied "SAT" user at the end of the transmit opportunities. Similarly, the expected unhappy users (labeled as "eUnHap") are approximately equal to the number of Hopeless (labeled as "HOP") users. However, the curves with feedback and Average strategy are more irregular, which is due to the changes of the strategy when receiving feedback regarding packet losses.

The user satisfaction result for Maximum strategy is presented in Fig. 2(b). All the users are expected to be initially happy, because the base station selected the maximum number of transmit opportunities as multicast value. Note that the user satisfaction level is the same regardless of whether the feedback is available or not.

\section{B. Allocation Policies Scenarios and Results}

In the multicast scenario, the same number of BL packets must be transmitted to each user. For this reason, the number of BL packets cannot be optimal for all users, but it is the result of a deal among different user requirements.

The results shows the evaluation of the effect of resource allocation policies with respect to goodput, packet delivery delay and quality versus $\lambda$ (The $\lambda$ is the threshold defining point regarding the amount of BL that must be transmitted). Fig. 3(a) shows that XLO performs near optimal, i.e., close to the Offline solution. The XLO approach takes into consideration the channel conditions of the user and jointly selects the BL or EL packet to be sent and the MCS to use, whereas the NXLO policy checks the need for BL/EL packets first and 


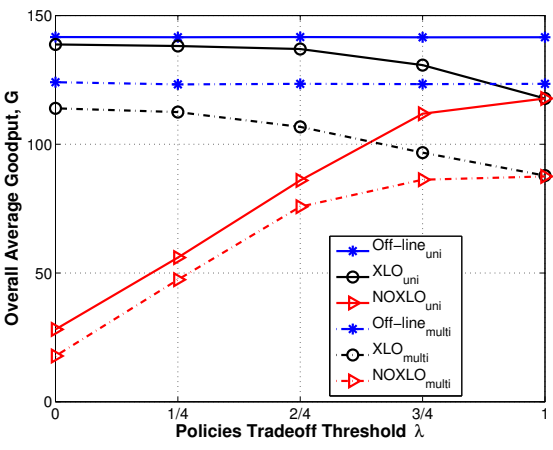

(a) $G$ vs. $\lambda$

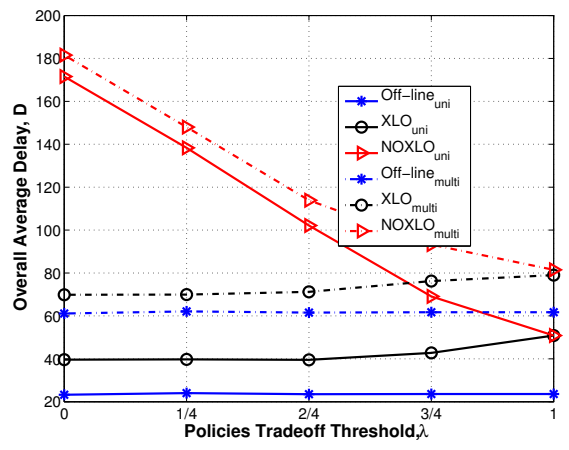

(b) $D$ vs. $\lambda$

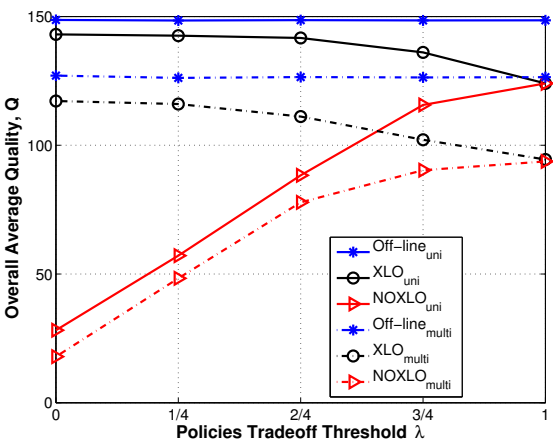

(c) $Q$ vs. $\lambda, \gamma=0.6$

Fig. 3: Multicast Performance evaluation versus the reservation parameter $\lambda$

then sequentially select the MCS. The performance is reported in terms of goodput, in Fig. 3(a).

On the other hand, Fig. 3(b) shows that XLO has lower packet delivery delay compared to NXLO and is almost as efficient as Offline. The quality $Q$ is a combination of goodput and delay and is illustrated in Fig. 3(c). The curves for quality results lie in between the goodput and delay curves, and the trend of quality curves can be varied by $\gamma$ value setting. Here we set $\gamma=0.6$.

Figs. 3 show the same trend of the curves in for unicast and multicast, although the performance of policies in multicast is slightly lower, because, the base station needs to transmit the same video content to all the users in the multicast session, for which the base station checks the channel conditions of all the users and tries to utilise aggregated channel information. Therefore, some of the users may be better served but few users may face quality degradation due to the compromise for other users. On average, the resulting quality is slightly decreased, even though the same trend persists.

\section{CONCLUSIONS}

To investigate the performance of PHY/Application crosslayer optimisation in a multicast environment, we consider an analytical model, where the channel is modelled by means of a Markov chain, whose states represent different channel qualities. We explored a cross-layer (PHY/Application) solution with respect to adaptive transmission rates for SVC layers. For this reason, we formulated a model to assess the performance of a cross-layer solution as opposed to a sequential selection of the two layers. We provide an optimisation framework to compute the optimum number of timeslots needed with respect to video quality maximisation and for evaluating the importance of feedback in multicast session for layered video contents delivery. We further evaluated via simulation the performance in terms of goodput, packet delivery delay and quality. We observed that the joint selection solution provides performance very close to the optimal/theoretical best performance(as provided by the Offline policy). An evolutionary work will involve investigating the effect of the number of users in a multicast group and how their joining and leaving can affect the quality of all users.

\section{ACKNOWLEDGEMENT}

Thanks to Dr. Leonardo Badia for guidance regarding analytical model presented here. Authors are funded by the European Commu- nity under its Seventh Framework Programme through the Reducing Internet Transport Latency (RITE) project (ICT-317700) and TimeIn project (213265-070) by Research Council of Norway. The views expressed are solely those of the authors.

\section{REFERENCES}

[1] "Cisco visual networking index: Global mobile data traffic forecast update, 2011-2016," Cisco, May 2012.

[2] S. Jelassi, G. Rubino, H. Melvin, H. Youssef, and G. Pujolle, "Quality of experience of VoIP service: A survey of assessment approaches and open issues," IEEE Communications Surveys and Tutorials,, vol. 14, no. 2, pp. $491-513,2012$.

[3] P. Li, H. Zhang, B. Zhao, and S. Rangarajan, "Scalable video multicast in multi-carrier wireless data systems," Proc. of IEEE ICNP,, pp. 141$150,2009$.

[4] Y. Ishibashi, Y. Tachibana, and S. Tasaka, "Responsiveness of layered multicast and feedback control for video traffic in the internet," Proc. of IEEE ICC,, vol. 2, pp. 846-852, 2000.

[5] L. Dounis, I. Politis, and T. Dagiuklas, "On the comparison of realtime rate control schemes for H.264/AVC video streams over IP-Based networks using network feedbacks," Proc. of IEEE ICC,, pp. 1-6, 2011.

[6] H. Schwarz, D. Marpe, and T. Wiegand, "Overview of the scalable video coding extension of the H.264/AVC standard," IEEE Trans. on Circuits and Systems for Video Technology, vol. 17, no. 9, pp. 1103-1120, 2007.

[7] H. Sohn, H. Yoo, W. De Neve, C. S. Kim, and Y. M. Ro, "Full-reference video quality metric for fully scalable and mobile SVC content," IEEE Trans. on Broadcasting, , vol. 56, no. 3, pp. 269-280, 2010.

[8] J. Widmer, A. Capalbo, A. Anta, and A. Banchs, "Rate allocation for layered multicast streaming with inter-layer network coding," Proc. of IEEE INFOCOM, pp. $2796-2800,2012$.

[9] A. Reis, J. Chakareski, A. Kassler, and S. Sargento, "Quality of Experience optimized scheduling in multi-service wireless mesh networks," Proc. of IEEE ICIP, pp. 3233-3236, 2010.

[10] C. N. Ververidis, J. Riihijarvi, and P. Mahonen, "Evaluation of Quality of Experience for video streaming over dynamic spectrum access systems," Proc. of IEEE WoWMoM, pp. 1-8, 2010.

[11] J. Kim, J. Cho, and H. Shin, "Resource allocation for scalable video broadcast in wireless cellular networks," Proc. of IEEE WiMob, 2005.

[12] J. Villalon, P. Cuenca, L. Orozxo-Barbosa, Y. Seok, and T. Turletti, "Cross-layer architecture for adaptive video multicast streaming over multirate wireless LANs," IEEE JSAC,, vol. 25, no. 4, May 2007.

[13] T. Andelin, V. Chetty, D. Harbaugh, S. Warnick, and D. Zappala, "Quality selection for dynamic adaptive streaming over HTTP with scalable video coding," Proc. of the 3rd Multimedia Systems Conference,, pp. 149-154, 2012.

[14] P. Li, H. Zhang, B. Zhao, and S. Rangarajan, "Scalable video multicast with adaptive modulation and coding in broadband wireless data systems," IEEE/ACM Trans. Netw., vol. 20, no. 1, pp. 57-68, 2012.

[15] I. Ahmed and L. Badia, "Analysis of management policies for multicast transmission of scalable video content in next generation networks," in Wireless Communications and Mobile Computing Conference (IWCMC), 2013 9th International, July 2013, pp. 1206-1211.

[16] A.R. Forsyth, Calculus of Variations. New York: Dover, 1960. 